\title{
A importância da aplicabilidade das normas técnicas de cabeamento estruturado de instituições de ensino superior visando a Educação Remota e a Distância (EAD)
}

\author{
The importance of the applicability of technical standards for structured cabling of higher \\ education institutions aiming at Remote and Distance Education (DL)
}

Recebido: 26/03/2021 | Revisado: 30/03/2021 | Aceito: 31/03/2021 | Publicado: 07/04/2021

\author{
André Ricardo Nascimento das Neves \\ ORCID: https://orcid.org/0000-0002-2911-5376 \\ Escola Superior Batista do Amazonas, Brasil \\ E-mail: aricardo.neves@gmail.com \\ Max Leandro de Araújo Brito \\ ORCID: https://orcid.org/0000-0003-2827-9886 \\ Universidade Federal do Rio Grande do Norte, Brasil \\ E-mail: maxlabrito@yahoo.com.br \\ Ricardo Shitsuka \\ ORCID: https://orcid.org/0000-0003-2630-1541 \\ Universidade Federal de Itajubá, Brasil \\ E-mail: rshitsuka@gmail.com \\ Dorlivete Moreira Shitsuka \\ ORCID: https://orcid.org/0000-0002-3282-4843 \\ Grupo de Pesquisas MEAC, Brasil \\ E-mail: dorlivetems@gmail.com \\ Caleb Shitsuka \\ ORCID: https://orcid.org/0000-0002-9813-0457 \\ Universidade Brasil, Brasil \\ E-mail: cashitsuka@gmail.com \\ Cláudio Boghi \\ ORCID: https://orcid.org/0000-0002-7974-6416 \\ Centro Universitário das Faculdades Metropolitanas Unidas, Brasil \\ E-mail: cboghi@uol.com.br \\ Rabbith Ive Carolina Shitsuka Risemberg \\ ORCID: https://orcid.org/0000-0003-1640-8898 \\ Private Practice, United States \\ E-mail: rabbith.ive@gmail.com
}

\begin{abstract}
Resumo
Atualmente, é comum observar as empresas fazendo grandes investimentos em switches, roteadores de alto desempenho, fontes processadoras não - redundantes e softwares. Contudo, desconhecem o meio físico, ou seja, o cabeamento estruturado. Para que o sistema funcione de forma adequada faz-se necessário que a estrutura seja montada de acordo com norma. O presente estudo tem o objetivo de apresentar um estudo de pesquisa-ação na qual se pode verificar a importância da rede de computadores em uma instituição de ensino, mostrando a diferença de desempenho devido investimento na infraestrutura em duas condições: uma de rede não guiada e outra de rede com fibra óptica. Os resultados da mudança no tipo de infraestrutura apontam para o atendimento da necessidade de velocidade por parte dos usuários professores e alunos. Sugere-se que as instituições de ensino superior que optam só por redes sem cabeamento procurem observar a satisfação dos usuários nesses tempos nos quais as redes de computadores tornam-se um dos principais itens da infraestrutura tecnológica das instituições de ensino superior.
\end{abstract}

Palavras-chave: Cabeamento; Ensino; Educação a Distância; Infraestrutura.

\begin{abstract}
Currently, it is common to see companies making large investments in switches, high performance routers, non redundant processor sources and software. However, they are unaware of the physical environment, that is, structured cabling. For the system to function properly, it is necessary for the structure to be assembled in accordance with the standard. The present study aims to present a case study of a computer network in a fictitious educational institution showing the difference in performance due to investment in infrastructure in two conditions: one with an unguided network and the other with an optical fiber network. The results of the change in the type of infrastructure point to
\end{abstract}


meeting the need for speed on the part of teachers and students. It is suggested that higher education institutions that choose only networks without cabling seek to observe user satisfaction in these times when computer networks become one of the main items of the technological infrastructure of higher education institutions.

Keywords: Cabling; Teaching; Distance Education; Infrastructure.

\section{Introdução}

No Brasil, as instituições de ensino e redes escolares, por meio do Parecer n. 19 do Conselho Nacional de Educação (CNE) aprova o ensino remoto até dezembro de 2021 em 06 de outubro de 2020 e, pela Resolução CNE/CP n. 2 de 10 de dezembro de 2020 podem trabalhar os processos educacionais remotamente enquanto durar a pandemia da Covid-19. Esta Resolução institui as Diretrizes Nacionais orientadoras para a implementação dos dispositivos da Lei n. 14040 de agosto de 2020 que estabelece normas educacionais excepcionais a serem adotadas pelos sistemas de ensino e redes escolares durante o estado de calamidade reconhecido pelo Decreto Legislativo n. 6 de 20 de março de 2020.

Para trabalhar remotamente, as instituições de ensino, que já possuíam o domínio dos processos educacionais a distância tiveram mais facilidade ao passo que outras que não possuíam esse expertise, tiveram que se adaptar buscando novas formas de trabalho dos processos educacionais por meio das ferramentas de tecnologia disponíveis na atualidade entre as quais, o celular, as redes de computadores e internet, os softwares de Educação a Distância e os meios de transmissão e mídias educacionais tradicionais por meio de papel, DVD e outros.

Barbosa e Shitsuka (2021) apresentam um exemplo do emprego de tecnologias digitais no ensino remoto do ensino fundamental e isso é característico dos tempos de pandemia do ano de 2020 nos quais houve a necessidade de adaptação do modo de trabalhar os processos educacionais entre alunos e professores e foi preciso se empregar as tecnologias disponíveis. É interessante que se enxerque que cada escola seja pública ou particular teve que se adaptar as novas condições, criando-se experiências próprias cujo compartilhamento pode ajudar a nuclear ideias e a criatividade necessária para que outros profissionais da educação repensem a melhoria dos seus recursos e o enriquecimento do saber e saber fazer, tanto sobre as formas de uso da tecnologia como também as formas de trabalhar os processos educacionais que no caso era para educação básica mas pode se estender também para outros níveis educacionais e outras tecnologias.

Como uma grande parcela da população usa celulares, WhatsApp, Internet e redes sociais, correio eletrônico e outras, essas tecnologias têm se mostrados úteis na comunicação entre os envolvidos nos processos educacionais.

Também como mostram Santos, Santos e Coelho (2021) no trabalho com as novas tecnologias como é o caso da internet das coisas e que é confirmada por Maciel, Leito e e Menezes em seus trabalhos com internet das coisas e outras tecnologias como redes sem fio de sensores como aponta Melo Jr et al, 2020) e, verifica-se que há uma quantidade cada vez crescente de dados e informações circulando pelas redes e há a necessidade que elas sejam seguras e capazes de trabalhar com essa quantidade de informações.

O trabalho remoto se beneficia das tecnologias de Educação a Distância que permite que professores e alunos possam participar dos processos educacionais mesmo havendo a distância física, por meio das tecnologias e softwares que permitem que ocorra a comunicação entre as partes envolvidas.

Atualmente, é comum observar as empresas fazendo grandes investimentos em infraestrutura de telecomunicações sem fio para internet, incluindo entre outros: switches, roteadores de alto desempenho, fontes processadoras não-redundantes e softwares sem conseguir alcançar o máximo rendimento uma vez que muitas, desconhecem o meio físico guiado, ou seja, o cabeamento estruturado. 
Objetivo do presente artigo é apresentar um estudo no qual demonstramos a importância do parque tecnológico incluindo seus ativos de telecomunicações para que a uma instituição de ensino tenha evolução positiva característica de uma organização que aprende e evolui para uma qualidade melhor.

\section{Metodologia}

A metodologia aponta os caminhos percorridas pelos pesquisadores para se chegar aos resultados e as conclusões como considera Pereira et al (2018). Concordando com os autores, procuramos trabalhar dentro de uma metodologia bem conhecida que é a do estudo de caso.

Pereira et al (2018), Thiollent (2011) e Tripp (2005) mostram que a pesquisa-ação é uma alternativa interessante para resolver problemas de organizações com a participação do envolvidos. Boghi et al. (2018) mostra um exemplo de aplicação da pesquisa ação muito embora voltado para área de saúde mas com princípios válidos para todas áreas do saber humano e Souza (2020) faz o emprego da pesquisa-ação na resolução de problemas relacionados ao projeto político pedagógico em uma escola envolvendo os professores. Santos et al. (2019) apresenta a resolução de problema em processo de ensino e de aprendizagem por meio do emprego de pesquisa-ação em processo educacional já Shitsuka, Shitsuka e Boghi (2018) apresentam a exemplo de resolução de dificuldades de tutoria na educação a distância por meio do emprego de pesquisa-ação. Também pode-se considerar que vivemos na era da informação e do conhecimento como afirmam Gama et al. (2016) e para esses autores, isso exige das pessoas e organizações conhecimento para saber utilizá-las e gerar resultados positivos para a organização. No entendimento dos autores do presente artigo, esse saber utilizar os conhecimentos e informações passa pela formação das pessoas que pode ocorrer por meio da educação infomal. Esta como consideram Pereira et al. (2019) quando trabalham a questão da didática na aprendizagem, pode ocorrer por meio de reuniões, envolvimento e comprometimento dos participantes no processo educacional em curso, seja ele formal (nos cursos de longa duração) ou informal que pode ocorrer na reunião de grupos sociais com objetivos comuns.

Seguindo as orientações dos teóricos acima mencionados e considerando a experiência dos autores os quais possuem experiência como coordenadores de curso e, atuam como avaliadores de cursos que necessitam de infraestrutura tecnológica para poder funcionar na modalidade distância, procurou-se focar na temática da infraestrutura tecnológica necessária ao bom funcionamento dos cursos nos tempos atuais seja devido à pandemia que faz com que muitas instituições tenham que trabalhar os processos educacionais remotamente ou, seja, evolução da legislação que atualmente permite que as instituições de ensino trabalhem com 40\% de Educação a Distância conforme Brasil (2019) e pelo avanço da Educação a Distância no Brasil em termos de quantidade de matriculas nesta modalidade em relação à modalidade presencial.

\section{Aspectos da Tecnologia na EaD em Cursos Superiores}

Segundo a Legislação vigente, por meio da Portaria n. 2117 de 06 de dezembro de 2019 que dispõe sobre a oferta de carga horária ofertados por Instituições de Educação Superior. Neste sentido, no seu artigo 7, determina que:

Art. $7^{\circ} \mathrm{Na}$ fase de Parecer Final dos processos de autorização de cursos presenciais, a possibilidade da oferta de carga horária a distância, até o limite de $40 \%$ da carga horária total do curso, além dos critérios estabelecidos pela Portaria Normativa MEC $n^{\circ}$ 20, de 21 de dezembro de 2017, está sujeita à obtenção, pelo curso, de conceito igual ou superior a três em todos os indicadores a seguir:

I - Metodologia;

II - Atividades de tutoria;

III - Ambiente Virtual de Aprendizagem - AVA; e

IV - Tecnologias de Informação e Comunicação - TIC. 
$\S 1^{\circ} \mathrm{O}$ não atendimento ao critério definido neste artigo ensejará o indeferimento do pedido de autorização do curso. (Brasil, 2019).

Por meio da Portaria se verifica que a tecnologia passa a ter um papel relevantes nos processos educacionais à distância.

Já segundo Brasil (2017) por meio do Decreto n. 9057 de 2017 é voltada para as condições de credenciamento, autorização e reconhecimento de cursos superiores na modalidade EaD e, nela há a também a exigência da metodologia e de recursos de infraestrutura física, tecnológica e de pessoal.

A legislação está coerente com a importância da EaD nos cursos superiores também tem aumentando em quantidade de cursos e matrículas de alunos nestes anos recentes em virtude do crescimento na quantidade de instituições que trabalham com EaD, Em 2019 houve 1,4 milhões de alunos a mais matriculados em graduações EaD em relação às presenciais (ABMES, 2019). Essses números confirmam os avanços e tendencias nesta modalidade educacional.

Os números mostram que a quantidade de matriculas na modalidade EaD tem crescido ano a ano a partir de 2013 e que em meados de 2018 superou a quantidade de matrículas em relação à educação presencial. Outro fato que chama a atenção é que a quantidade de matrículas na educação presencial não para de diminuir enquanto a da educação a distância continua em crescimento conforme os dados apresentados.

De acordo com INEP (2020):

A tendência de crescimento do ensino a distância $(\mathrm{EaD})$ se confirma, a cada ano, na educação superior brasileira. Em $2019,63,2 \%$ (10.395.600) das vagas ofertadas foram nessa modalidade, entre as 16.425 .302 vagas disponíveis para o nível de ensino, no total. Os dados fazem parte dos resultados do Censo da Educação Superior 2019, divulgados pelo Instituto Nacional de Estudos e Pesquisas Educacionais Anísio Teixeira (Inep) e pelo Ministério da Educação (MEC). O censo mostra ainda que, em 2019, o número de ingressantes em cursos de EaD ultrapassou a quantidade de estudantes que iniciaram a graduação presencial, na rede privada. Ao todo, 50,7\% (1.559.725) dos alunos que ingressaram em instituições privadas optaram por cursos de EaD. Em contraponto, 49,3\% (1.514.302) dos estudantes escolheram ingressar na educação superior de modo presencial.

Como a quantidade de alunos está em crescimento na educação a distância, também com o trabalho em regime Remoto devido à pandemia mundial causada pela COVID-19 e, devido à legislação que têm evoluído para os cursos a distância, torna-se interessante que haja o trabalho nesta modalidade educacional. Sendo assim, para que o ensino seja remoto ou a distância, ocorra ao contento dos alunos e profissionais do ensino, torna-se interessante que ocorra com uma infraestutura tecnológica adequada. Uma das principais tecnologias na telecomunicação por meio de computadores é a do cabeamento que pode ser não guiado por meio de ondas de rádio ou guiado por meio de cabeamento físico que pode ser estruturado ou não estruturado como considera Tanenbaum (2011).

As tecnologias mais lentas atualmente em termos de velocidade de transmissão e compartilhamento de dados são as das redes de meios não guiados ou redes não cabeadas e as mais velozes são as redes guiadas que funcionam com cabeamento estruturado óptico ou cabos de Par trançado nas categorias atuais.

\section{Pesquisa-Ação e Discussão}

Em uma instituição denominada XYZ, no primeiro semestre de 2019, antes da época da pandemia, na qual a rede funciona sem cabeamento ou por meio não guiado observando a insatisfação dos envolvidos que sempre faziam suas reclamações por meio dos levantamentos realizados pela Comissão Própria de Avaliação (CPA) da instituição. A comunicação feita majoritariamente por meios não guiados de comunicação, comprometendo assim, a qualidade e a confiabilidade para transmissão dos dados e o pleno funcionamento de seus sistemas. As notas atribuídas de zero a dez eram muito baixas para a 
rede que tinha média por volta de 3 . Houve reuniões com alunos, pessoal de TI, coordenação e levantou-se a proposta conjunta de troca da rede para uma rede cabeada de fibra óptica que é a mais veloz de todas.

Analisando os laboratórios, verificou-se que não havia infraestrutura adequada e suficiente para comportar os equipamentos ativos e passivos da rede atual, bem como os procedimentos que não condizem com as normas de cabeamento, dificultando o desempenho da rede, gerenciamento e até a perda de dados na rede. Ainda em meados de 2019 a instituição já prevendo a necessidade de ter uma infraestrutura tecnológica adequada como considera Tanenbaum (2011) e, aos planos de EaD para atender ao avanço da EaD por meio de Brasil (2017) no seu Decreto 9057 de 2017. Houve uma "aposta" da faculdade no sentido de atender às reclamações dos alunos e ir ao encontro de uma infraestrutura tecnologia mais estável, rápida, segura e que atendesse melhor os alunos.

No levantamento realizado no final de 2019 os alunos mostraram-se satisfeitos com a nova infraestrutura disponibilizada aos alunos e, que em nova avaliação mostrou que a grande maioria, mais de $80 \%$ atribuiu nota maior que 7 (sete) à tecnologia de informação da instituição.

Outro aspecto importante é que os cursos superiores e instituições de ensino no Brasil passam por avaliações externas de credenciamento periodicamente. Estas têm a finalidade verificar as condições de estrutura de oferta e a realização dos trabalhos em conformidade com seu projeto pedagógico e a estrutura física. As organizações desde março de 2020 quando o mundo entrou numa pandemia avassaladora tiveram que adquirir a expertise do ensino a distância. Isso aumentou significativamente os processos e pedidos de credenciamento de cursos ou instituições nessa modalidade. Atualmente, é comum observar as empresas fazendo grandes investimentos em switches, roteadores de alto desempenho, fontes processadoras não - redundantes e softwares. Contudo, desconhecem o meio físico, ou seja, o cabeamento estruturado. Para que o sistema funcione de forma adequada faz-se necessário que a estrutura seja montada de acordo com norma. As redes computacionais não podem ser projetadas de forma limitada, mas expansiva, de forma que esta consiga acompanhar o desenvolvimento tecnológico configurando-se em uma rede futura que esteja pronta para receber novas tecnologias.

A questão tecnológica é importante, uma vez que mesmo atualmente, é comum observar as empresas fazendo grandes investimentos em switches, roteadores de alto desempenho, fontes processadoras não - redundantes e softwares. Contudo, desconhecem o meio físico, ou seja, o cabeamento estruturado. Para que o sistema funcione de forma adequada faz-se necessário que a estrutura seja montada de acordo com norma. As redes computacionais não podem ser projetadas de forma limitada, mas expansiva, de forma que esta consiga acompanhar o desenvolvimento tecnológico configurando-se em uma rede futura que esteja pronta para receber novas tecnologias. Uma das formas é por meio do emprego da normatização. Um exemplo geral disso, é o emprego das normas ABNT (2002a) que é para citações em textos, que é a NBR 10520 e que funciona em conjunto com a ABNT(2002b) que é a NBR 6023 que é de referências. Para nós, há uma agregação de valor ao artigo devido às citações e referências conforme a norma, uma vez que a informação torna-se possível de ser recuperado dentro de uma condição normatizada. De modo semelhante, há o emprego de normas na área da Tecnologia de Informação e está também agrega valor trazendo inovação. Este trabalho apresenta os resultados acerca da aplicabilidade da NBR 14565:2013, “Cabeamento estruturado para edifícios comerciais e data centers”. O projeto de instalação em uma rede computacional tem como principal objetivo, a instalação e ampliação da rede de dados com uma infraestrutura montada em cabeamento de rede lógica, onde usamos eletrodutos e eletrocalhas de modo organizado, identificado e seguro. No cabeamento estruturado fazemos uso de tecnologias atuais para assegurar o uso racional e seguro como é o caso da Fast Ethernet e Gigabit Ethernet. Com esse sistema montado, conquistou-se a qualidade dos serviços executados, a compatibilidade com os sistemas atuais e futuros além da interoperabilidade dos sistemas. 


\section{Considerações Finais}

Este trabalho demonstrou a relevância da estrutura cabeamento estruturado seja por meio de cabeamento óptico ou metálico em redes computacionais em uma Instituição de Ensino superior utilizando cabos específicos para atender ao planejamento traçado de todo o projeto de instalação elétrica de um computador ao outro, ou até mesmo entre vários computadores, a Intranet e sucessivamente a internet.

Desta forma, podemos assegurar que o uso de cabos par trançado blindado e par traçado não blindado bem como, cabo tipo fibra óptica, são os mais seguros e aconselháveis para se ter um sistema de cabeamento estruturado mais seguro, confiável e duradouro e longe de obter interferências eletromagnéticas, o que de certa forma, só vem a prejudicar a transmissão de dados e a modulação das informações trocadas entre os computadores.

Por intermédio do estudo realizado é possível afirmar que todo sistema de cabeamento estruturado hoje para que seja considerado mais seguro, prático e com velocidade constante e sem gargalos causados por interferências eletromagnéticas, para isto deve-se nortear todo o sistema de montagem do cabeamento observando parâmetros técnicos e normatizados contidos na norma brasileira NBR 14565:2013.

Como o presente estudo foi realizado anteriormente à pandemia, torna-se interessante que após após a passagem desta fase se realizem novos estudos tanto no sentido de buscar mais avanços nas tecnologias de redes como também em relação à satisfação dos usuários dos serviços das redes uma vez que esses usuários estão cada vez mais exigentes uma vez que muitos passaram por teletrabalho, serviço remoto e aulas remotas e agora podem apresentar um novo patamar em suas exigências e isso é um fato interessante em se verificar nos próximos estudos e em muitas instituições.

\section{Referências}

ABMES. Ensino a Distância supera presencial, e faculdades privadas adaptam negócios. Publicado no website da Associação Brasileira de Mantenedoras do Ensino Superior (ABMES) em 2019. Recuperado de: https://abmes.org.br/noticias/detalhe/3601/ensino-a-distancia-supera-o-presencial-e-faculdades-privadasadaptam-negocios. Acesso 13 março de 2021.

ABNT NBR 14565:2013. Cabeamento estruturado para edifícios comerciais e data centers. Esta Norma especifica um sistema de cabeamento estruturado para uso nas dependências de um único ou um conjunto de edifícios comerciais em um campus, bem como para a infraestrutura de cabeamento estruturado de data centers. Ela cobre os cabeamentos metálico e óptico. Associação Brasileira de Normas Técnicas (ABNT), 2013.

ABNT NBR 6023. Informação e documentação - Referências - Elaboração. Associação Brasileira de Normas Técnicas (2002).

ABNT NBR 10520. Informação e documentação - Citações em documentos - Apresentação. Associação Brasileira de Normas Técnicas (2002). BARBOSA, R. A. S.; SHITSUKA, R. Uso de tecnologias digitais no ensino remoto de alunos da educação infantil e anos iniciais do ensino fundamental: relato de experiência. e-Acadêmica, v. 1, n.1, e12, 2020(CC BY 4.0). Recuperado de: https://eacademica.org/eacademica/article/view/12/12.

BOGHI, C.; SHITSUKA, D. M.; SHITSUKA, C. D. W. M.; RISEMBERG, R. I. C. S. Investigación-acción en estudiantes con dificultades psicosociales a través de terapias alternativas. Research, Society and Development, v. 7, n. 6, p. e1276345, 2018. DOI: 10.17648/rsd-v7i6.345. Disponível em: https://rsdjournal.org/index.php/rsd/article/view/345.

BRASIL. Portaria No 2.117, de 6 de dezembro de 2019. Ministério da Educação. Recuperado de: https://www.in.gov.br/en/web/dou/-/portaria-n-2.117-de-6de-dezembro-de-2019-232670913. Acesso em: 12 março 2021.

BRASI. Decreto $N^{o} 9057$ de 25 de maio de 2017. Regulamenta o Artigo 80 da Lei n. 9394 de 20 de dezembro de 1996, que estabelece as diretrizes e bases da educação nacional. Presidência da Repúbilca. Recuperado de: http://www.planalto.gov.br/ccivil_03/_ato2015-2018/2017/decreto/d9057.htm.

BRASIL. Parecer n. 19 do Conselho Nacional de Educação (CNE). Aprova o Ensino Remoto até dezembro de 2021 e, 06 de outubro de 2020 . Ministério da Educação. Conselho Nacional de Educação (CNE). 2020.

GAMA, M. X. B.; LAMOGLIA, C. V. A.; COSTA, W. L.; SAID, R. A.; SHITSUKA, D. M.; JUNIOR, D. M. P. Leadership in the Age of Information and Knowledge in companies. Research, Society and Development, v. 3, n. 1, p. 02-18, 2016. DOI: 10.17648/rsd-v3i1.37. Recuperado de: https://rsdjournal.org/index.php/rsd/article/view/37.

INEP. Ensino a distância se confirma como tendência. Publicado no website do Instituto Nacional de Estudos e Pesquisas Educacionais Anísio Teixeira (INEP) em 2020. Recuperado de: https://www.gov.br/inep/pt-br/assuntos/noticias/censo-da-educacao-superior/ensino-a-distancia-se-confirma-comotendencia. Acesso 24 março de 2021.

MACIEL, K. A.; LEITE, D. M.; MENEZES, J. W. M. Prototype of domestic application for the monitoring of water distribution systems based on internet of things. Research, Society and Development, v. 9, n. 10, p. e2059108274, 2020. DOI: 10.33448/rsd-v9i10.8274. Disponível em: https://rsdjournal.org/index.php/rsd/article/view/8274. 
MELO JUNIOR, G. de; OLIVEIRA FILHO, R. M. de; VIEIRA, S. L.; MACEDO, S. O. de ; FURRIEL, G. P.; SILVA, B. C. R. . Development of a wireless sensor network using ZigBee protocol for data monitoring and actuator activation. Research, Society and Development, v. 9, n. 10, p. e2729108651, 2020. DOI: 10.33448/rsd-v9i10.8651. Disponível em: https://rsdjournal.org/index.php/rsd/article/view/8651.

PEREIRA, A. S. et al. Metodologia da pesquisa cientifica. Santa Maria: UAB/NTE/UFSM, 2018. Recuperado de: https://www.ufsm.br/app/uploads/sites/358/2019/02/Metodologia-da-Pesquisa-Cientifica_final.pdf.

SANTOS, A. M. dos .; SANTOS, G. S.; COELHO, A. S. Internet oh Things: Use of Arduino to combat electric waste in IFMA Campus São João dos Patos. Research, Society and Development, v. 10, n. 3, p. e231035794, 2021. DOI: 10.33448/rsd-v10i3.5794. Recuperado de: https://rsdjournal.org/index.php/rsd/article/view/5794.

SANTOS, F. W. C. dos; ROSA, A. H.; AQUINO, F. J. A. de; OLIVEIRA, J. do N.; MACHADO, A. V. de S. Identification of difficulties in the teaching learning process through the program PIBID (Institutional Program of Scholarship Initiation to Teaching): a case study on the difficulties encountered in teaching the physics discipline of a public school in the city of Parnaíba. Research, Society and Development, v. 8, n. 8, p. e34881234, 2019. DOI: 10.33448/rsd-v8i8.1234. Disponível em: https://rsdjournal.org/index.php/rsd/article/view/1234.

SHITSUKA, R.; SHITSUKA, D.M.; BOGHI, C. Pesquisa-ação na Superação de Dificuldades de Acompanhamento de Curso de Pósgraduação a "Lato Sensu" a Distância por meio de Apoio da Tutoria. Rev. Bras. Aprend. Aberta. ABED. 2018; 1: e71.

SOARES, A. S. et al. Didática geral [recurso eletrônico. Santa Maria, RS : UFSM, NTE, 2019. [free e-book]. ISBN 978-85-8341-254-0. Recuperado de: https://repositorio.ufsm.br/bitstream/handle/1/19110/Curso_Lic-Ed-Esp_Did\%C3\%A1tica-Geral.pdf?sequence=1\&isAllowed=y

SOUZA, L. P. de . Democratic management and the Pedagogical Political Project: practices and contradictions of the school reality in Goianésia do Pará/PA. Research, Society and Development, v. 9, n. 12, p. e1309129692, 2020. DOI: 10.33448/rsd-v9i12.9692. Disponível em: https://rsdjournal.org/index.php/rsd/article/view/9692.

TANENBAUM, A. S.; WETHERALL, D. J. Redes de computadores. 5.ed. São Paulo: Pearson, 2011.

THIOLlENT, M. Metodologia da pesquisa-Ação. 18. ed. São Paulo: Cortez, 2011.

TRIPP, D. Pesquisa-ação: uma introdução metodológica. Educação \& Pesquisa, São Paulo, v. 31,n. 3,p. 443-466,set./dez.2005. 